\title{
THE AGES OF ELLIPTICAL GALAXIES IN A MERGER MODEL
}

\author{
G. KAUFFMANN \\ Max-Planck-Institut für Extraterrestrische Physik \\ D-85740 Garching, Germany
}

\section{Introduction}

There have long been two competing views on the formation history of the ellipticals galaxies we see today. One is that most of the stars in presentday galactic bulges and ellipticals were produced during a relatively short, early phase of intense star formation at high redshift. The second view is that elliptical galaxies are relative latecomers, having been produced as the result of the merging of disk galaxies drawn together by gravity as their surrounding dark matter halos coalesced.

In recent years, the second view has come to enjoy increasing popularity. Detailed numerical simulations have shown that mergers between two spiral galaxies of comparable mass lead to the production of spheroidal merger remnants with physical characteristics such as density profiles, gravitational radii, mean velocity dispersions and surface brightnesses that are quite comparable to to observed ellipticals (see Barnes \& Hernquist 1992 for a review). In addition, observational evidence has begun to accumulate that mergers and interactions are rather common in the universe, particularly at higher redshift. Hubble Space Telescope images indicate that at redshifts $\simeq 0.4,25-30 \%$ of all galaxies show disturbed morphologies (Griffiths et al 1995). At lower redshifts, almost all the most luminous IRAS galaxies have turned out to be interacting systems, leading to the conclusion that the merging process may fuel very intense bursts of star formation.

The fact remains, however, that the stellar populations of present-day elliptical galaxies appear to be rather homogeneous. The colours, mass-tolight ratios and $\mathrm{Mg}_{2}$ absorption strength of ellipticals are correlated only with their central velocity dispersions, and the scatter about these relations appears to be surprisingly small. Bower, Lucey \& Ellis (1992) show that the intrinsic scatter about the U-V and V-K colour-magnitude relations for early-type galaxies in the Coma and Virgo clusters is less than 0.05 
mag. This leads to the conclusion that elliptical galaxies must have formed the bulk of their stars at redshifts greater than 2, or else the observed homogeneity in the colours would require a very precise synchronization of formation epochs and star formation histories for these galaxies. Likewise, Bender, Burstein \& Faber (1993) find a very tight relationship between the strength of the $\mathrm{Mg}_{2}$ index at the centre of ellipticals and their central velocity dispersions, $\sigma$.

The tightness of these limits calls into question the tenability of the merger model, as merging might be expected to mix together stellar populations of quite disparate ages (Renzini 1994). In addition, a starburst accompanying the merging event would increase the amount of light contributed by young stars. However, detailed predictions have not yet been made for any realistic theory of galaxy formation via merging. Here, I present results calculated for a $b=1.5$ cold dark matter (CDM) universe using semi-analytic techniques. In this model, elliptical galaxies are produced when two progenitor galaxies of roughly equal mass merge with each other. Spiral galaxies are formed in a two-step process. Two galaxies of equal mass must first merge with each other to form the bulge component. Gas from the surrounding dark matter halo then cools and settles to form a new disk. I first consider the stellar populations of elliptical galaxies in clusters. I also extend the analysis to the stellar populations of field elliptical galaxies. Finally, I make some predictions for the scatter in the colours of cluster elliptical galaxies at high redshift.

\section{Semianalytic Models of Elliptical Galaxy Formation}

The semi-analytic models we employ are described in detail in Kauffmann, White \& Guiderdoni (1993, hereafter KWG) and Kauffmann \& White (1993). Application of the model to the evolution of the galaxy population in clusters at high redshift is discussed in Kauffmann (1995).

To summarize:

I use an algorithm based on an extension of the Press-Schechter theory due to Bower (1991) and Bond et al (1991) to generate Monte Carlo realizations of the merging paths of dark matter halos from high redshift until the present. Dark matter halos are modelled as truncated isothermal spheres and it is assumed that as the halo forms, the gas relaxes to a distribution that exactly parallels that of the dark matter. Gas then cools and condenses onto a central galaxy at the core of each halo. Star formation and feedback processes take place as described in KWG. In practice, star formation in central galaxies takes place at a roughly constant rate of a few solar masses per year, in agreement with the rates derived by Kennicutt (1983) for normal spiral galaxies. 
At a subsequent redshift, a halo will have merged with a number of others, forming a new halo of larger mass. All gas which has not already cooled is assumed to be shock heated to the virial temperature of this new halo. This hot gas then cools onto the central galaxy of the new halo, which is identified with the central galaxy of its largest progenitor. The central galaxies of the other progenitors become satellite galaxies, which are able to merge with the central galaxy on a dynamical friction timescale. If a merger takes place between two galaxies of roughly comparable mass, the merger remnant is labelled as an "elliptical" and all cold gas is transformed instantaneously into stars in a "starburst".

Note that the infall of new gas onto satellite galaxies is not allowed, and star formation will continue in such objects only until their existing cold gas reservoirs are exhausted. Thus the epoch at which a galaxy is accreted by a larger halo delineates the transition between active star formation in the galaxy and passive evolution of its stellar population. The stellar populations of elliptical merger remnants in clusters hence redden as their stellar populations age Central galaxy merger remnants in the "field" are able to accrete new gas in the form of a disk to form a "spiral" galaxy consisting of both a spheroidal bulge and a disk component. As demonstrated in KWG, this picture is able to account for the observed numbers and luminosity distributions of galaxies of different morphologies, both in clusters and in lower-density environments.

The spectrophotometric models of Bruzual \& Charlot (1993) are used to translate the predictions of the models into observed quantities such as magnitudes and colours, which may be compared directly with the observational data. Results are presented for a $b=1.5 \mathrm{CDM}$ universe with $\Omega=1$ and $H_{0}=50 \mathrm{~km} \mathrm{~s}^{-1} \mathrm{Mpc}^{-1}$.

\section{The Ages and Colours of Elliptical Galaxies}

Figure 1 shows the $\mathrm{V}$ luminosity-weighted mean stellar age of the galaxies in a cluster of $10^{15} M_{\odot}$. The classification into morphological type is based on the B-band disk-to-bulge ratio of the galaxy, as given in Table 3B of Simien \& de Vaucouleurs (1986). As can be seen, the stellar populations of spiral galaxies span a very wide range in age, but those of early-type galaxies are confined to ages between 8 and 12.5 Gyr. For our adopted cosmological parameters, this means that the bulk of stars in elliptical and SO galaxies were formed at redshifts exceeding 1.9.

The age distribution of early-type galaxies found in halos of mass $10^{13} M_{\odot}$ is plotted in figure 2. These galaxies either occur in small groups, or have no companion of comparable luminosity, and thus can be regarded as "field ellipticals". In order to get a a sizeable sample of these objects, it was nec- 


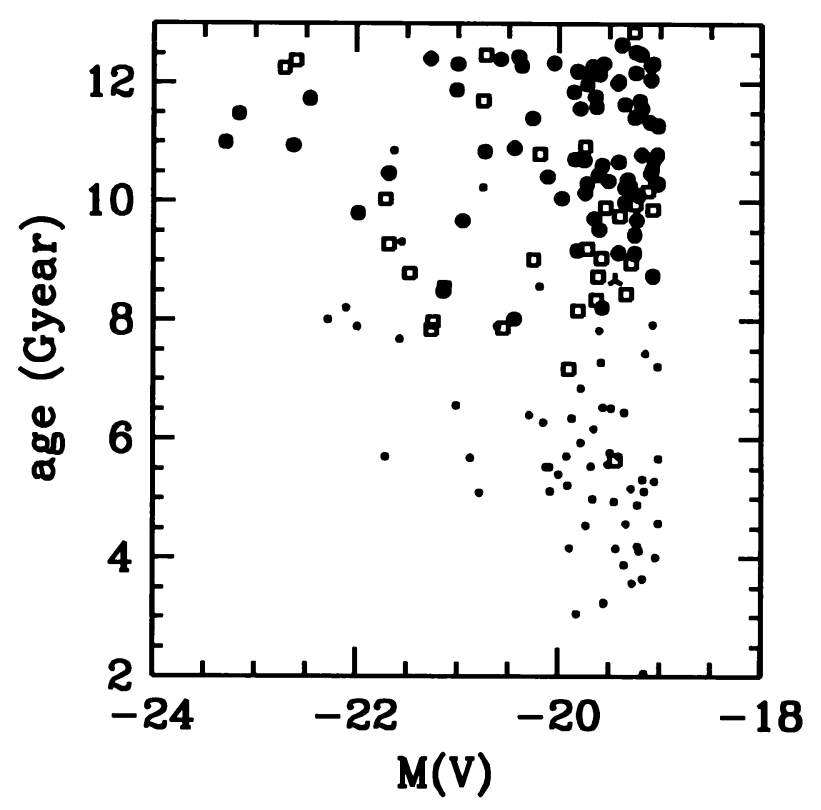

Figure 1. The V-luminosity weighted mean stellar ages of galaxies in a cluster of mass $10^{15} M_{\odot}$. Large filled circles represent ellipticals, open squares are SOs, small filled circles are spirals and 3-pronged pinwheels are elliptical galaxies that were formed by a major merger in the past 1.5 Gigayears.

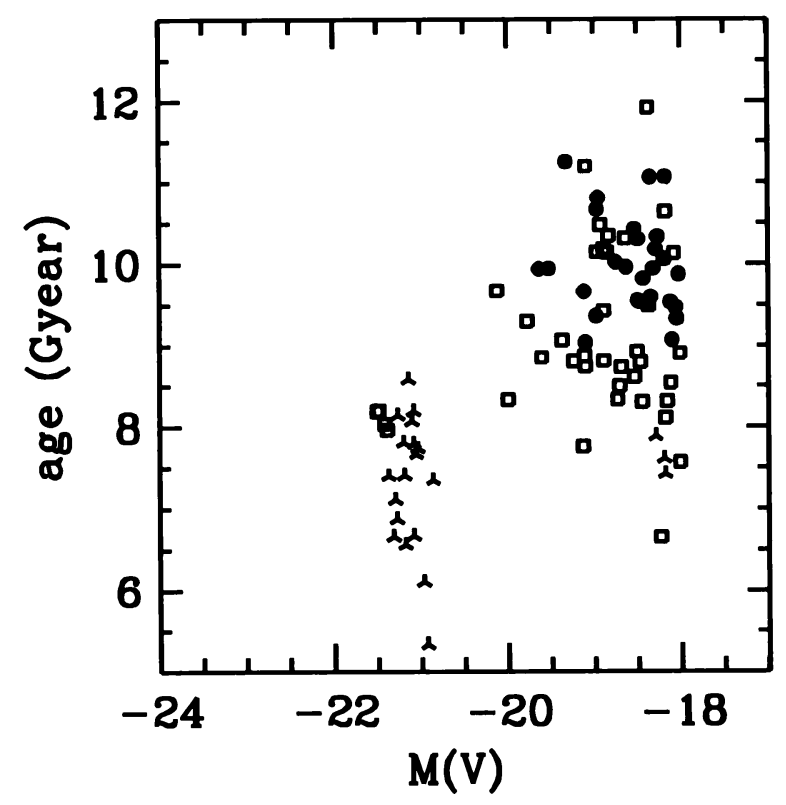

Figure 2. The mean ages of the early-type galaxies in halos of mass $10^{13} M_{\odot}$. Symbols are as in figure 1. 

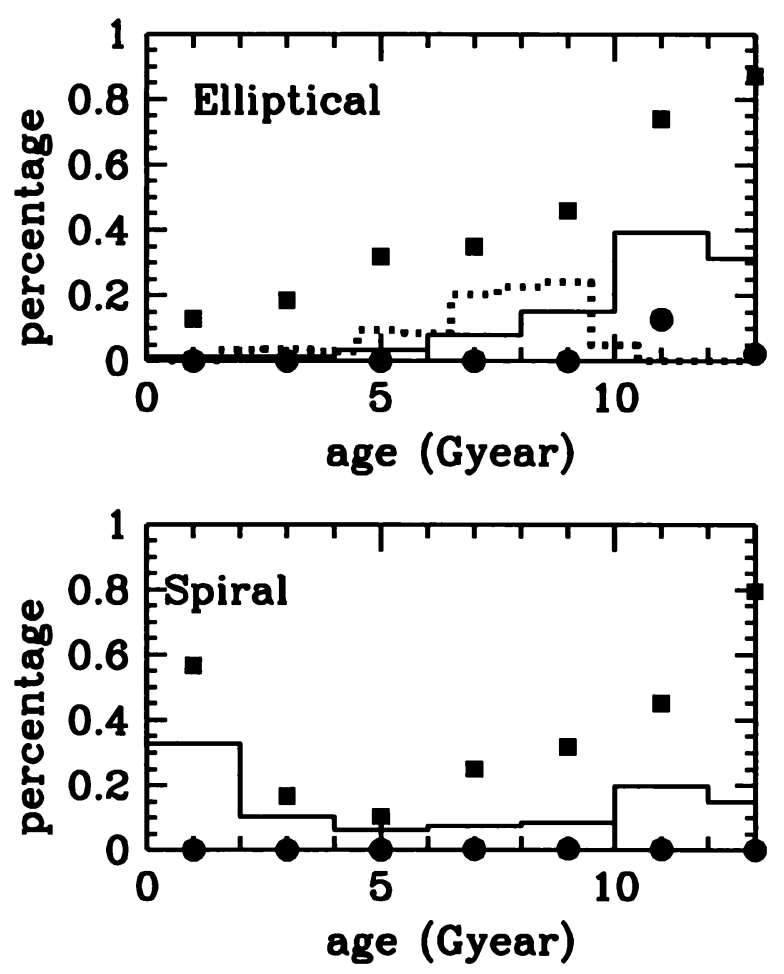

Figure 3. The fraction of the total V-band light of cluster ellipticals and spirals contributed by stars which form at a given epoch. The solid line is the average star formation history. The squares and circles represent the maximum and minimum contributions to the total light for any galaxy at that epoch. The dotted line is a histogram of the percentage of elliptical galaxies that experienced their last major merger at a given epoch.

essary to combine the populations of 100 such low-mass halos. As can be seen, the field elliptical population splits up into two distinct classes. The faint field ellipticals have stellar ages comparable to early-type galaxies in clusters. There is also a population of bright field ellipticals that have much younger stellar populations (mean ages between 5 and 8 Gigayears). These bright galaxies are all central galaxies and thus are still in the process of accreting cold gas. They are also mostly recent mergers. The fact that we see these galaxies as ellipticals is simply because they have not yet had the time to accrete a new disk following their last big merging event.

Figure 3 is a histogram of the fraction of the total V-band light of a cluster elliptical or spiral galaxy contributed by stars which form at a given redshift. A large fraction of the V-band light of spirals comes from stars that formed in the past $5 \mathrm{Gyr}$, whereas on average, this contribution is negligible in ellipticals. The dotted line in the top panel of figure 3 illustrates the epoch at which elliptical galaxies typical underwent their last major 

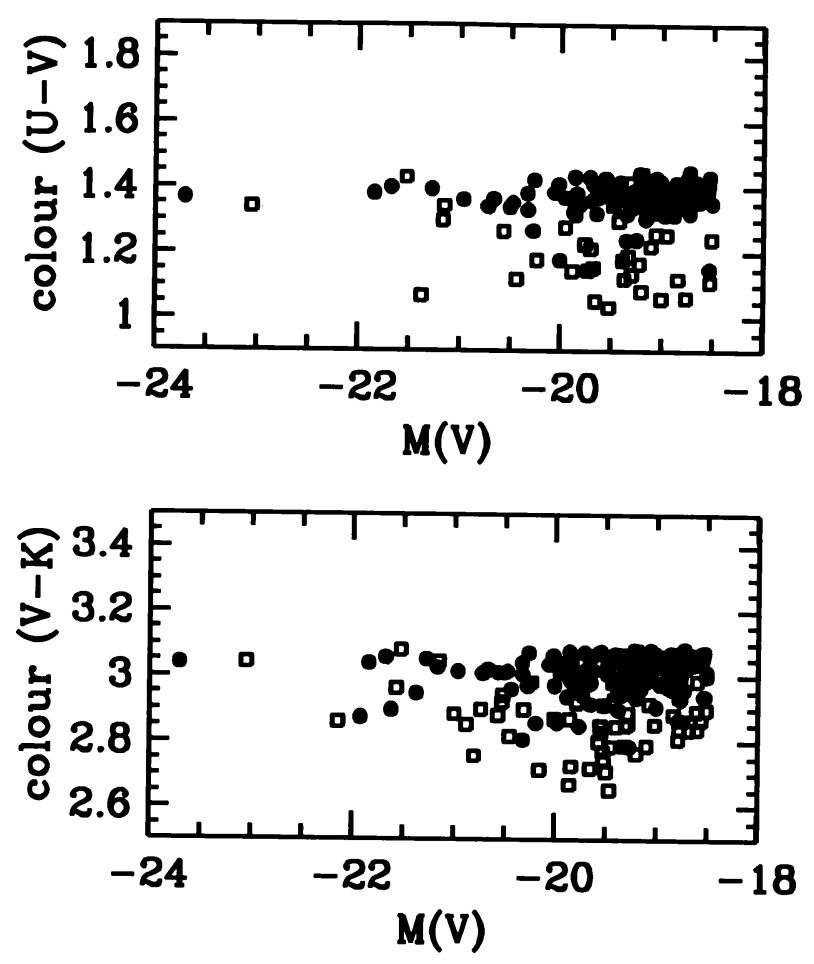

Figure 4. The distribution in U-V and V-K colour of early-type galaxies in a cluster of $10^{15} M_{\odot}$. Filled circles are ellipticals and open squares are SOs.

merging event. As can be seen, most ellipticals are formed by a merger that occurred between 5 and $10 \mathrm{Gyr}$ ago. It is also apparent from this diagram that a substantial fraction of the stars in an elliptical galaxy may be formed before the merging event that actually causes the transformation in morphology. Star formation may take place at a constant rate in the two spiral progenitors of an elliptical for several gigayears before they are able to merge. It is thus important to distinguish clearly what is meant by "the formation epoch of ellipticals". It can be defined either as the epoch when the bulk of the stars were formed or as the epoch when the merging event took place.

Finally, in figure 4, I have used the evolutionary synthesis models of Bruzual \& Charlot (1993) to generate a scatterplot of the U-V and V-K colours of early-type galaxies in clusters. In U-V, the average colour of an elliptical is 1.37 with a rms standard deviation, $\sigma$, of 0.034 . In V-K, the mean is 3.01 and $\sigma$ is 0.045 . If $\mathrm{SO}$ galaxies are included, the rms increases to $\simeq 0.07$. Note that the colour- magnitude relation is flat. As shown in figure 1 , cluster elliptical galaxies of all luminosities have roughly the same mean 
stellar age, so any trend in colour would have to result from metallicity differences between galaxies of different masses. The rms scatter in the colour of the model ellipticals is well within the upper limit for the intrinsic scatter in the colour of Coma and Virgo ellipticals quoted by Bower, Lucy \& Ellis (1992).

\section{Ellipticals in Clusters at High Redshift}

Present-day elliptical galaxies contain the oldest stars in the universe. The repair of HST permits the direct identification of elliptical and SO galaxies at significant cosmic depth. The hope is, therefore, that studies of the stellar populations of ellipticals at early epochs will place strong constraints on cosmology.

Ellis (1995) has measured the rest-frame U-V colours of elliptical galaxies in the cluster $0016+16$ at $z=0.54$ and finds a rms scatter of 0.07 . In figure 5 , model predictions for the $\mathrm{U}-\mathrm{V}$ colours of cluster ellipticals are shown at a series of redshifts. There are two trends that should be noted. Firstly, the "red envelope" of the distribution shifts bluewards with increasing redshift. The magnitude of this shift is consistent with what one would expect from the passive evolution of a 12 Gyr old burst, as given by the models of Bruzual \& Charlot (1993). Secondly, the scatter in the colour distribution increases with redshift and most of this increase arises from a growing population of galaxies which have merged in the 1.5 Gyr prior to the epoch of observation. Note, however, that the scatter is not dramatically large until redshifts in excess of 1 . This may be understood as follows. As pointed out by Charlot \& Silk (1994), the colours of stars formed in a short burst evolve very rapidly for the first 3-4 Gyr. After that, the evolution slows down and the colours redden only gradually over time. So long as most of the stars in the elliptical population of a cluster are formed 3-4 Gyr prior to the time the cluster is observed, no great conspiracy is required to produce a small scatter in the colours. At redshifts greater than 1, this is no longer possible (assuming $\Omega=1$ and $H_{0}=50 \mathrm{~km} \mathrm{~s}^{-1} \mathrm{Mpc}^{-1}$ ) and the scatter becomes very large. Spectroscopic age indicators may turn out to be more sensitive tests of cosmology.

\section{Discussion \& Conclusions}

Two conclusions follow from this analysis. One is that star formation in cluster ellipticals formed by mergers occurs at high enough redshifts so that the predicted scatter in the colour- magnitude relation of these systems falls within observational bounds. That ellipticals in clusters form at high redshift should come as no surprise. For a random Gaussian initial density field, it is well-known that the redshift of collapse of density peaks on 

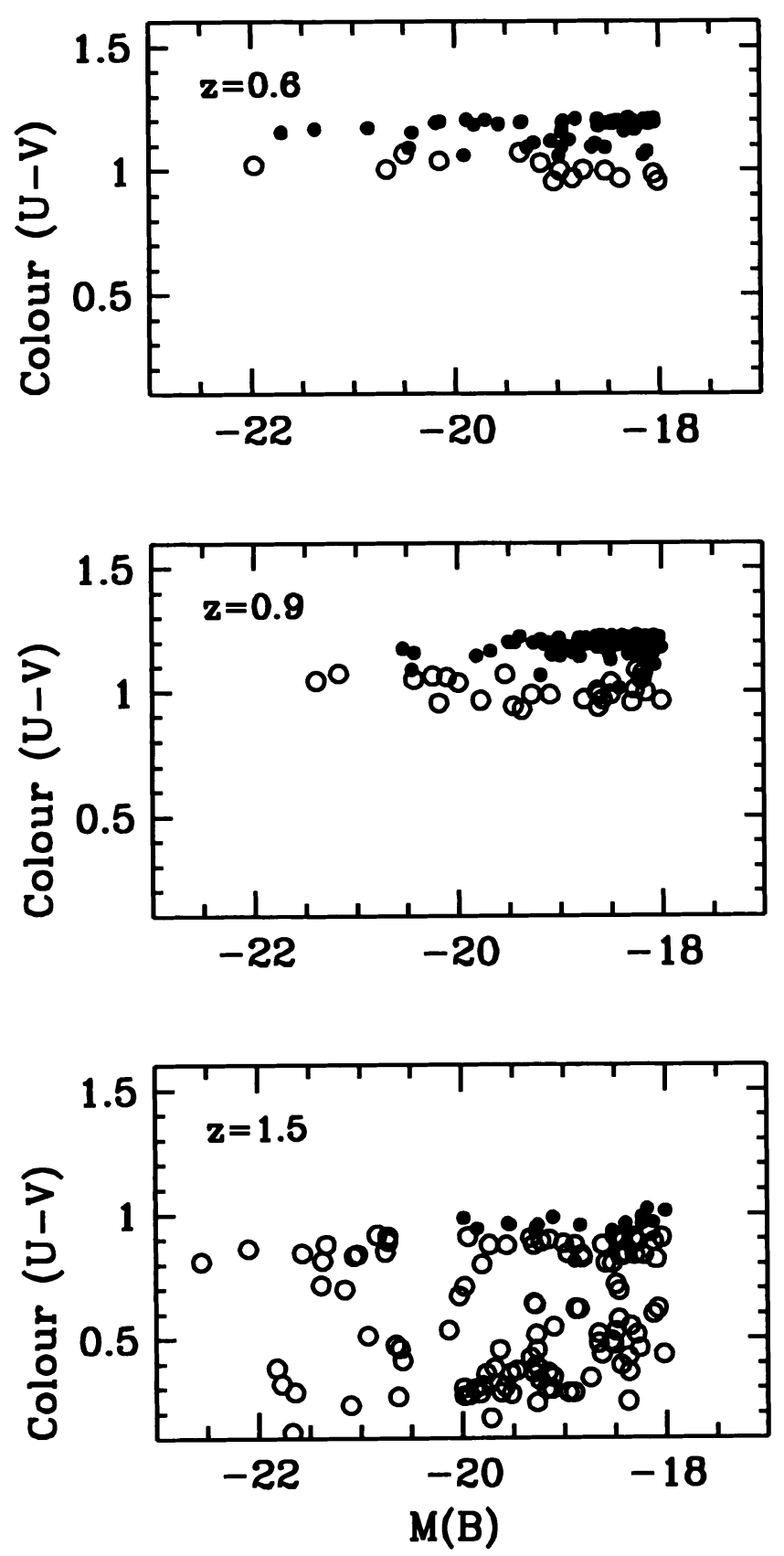

Figure 5. Rest-frame U-V colour-magnitude relations for elliptical galaxies in clusters of $10^{15} M_{\odot}$ observed at $\mathrm{z}=0.6,0.9$ and 1.5. Large open circles denote ellipticals that have been formed by a major merging event in the past $1.5 \mathrm{Gyr}$. 
galaxy scales is boosted by the presence of the surrounding, larger-scale overdensity destined to collapse to form the cluster at $\mathrm{z}=0$ (Bardeen et al 1986). Galaxies forming in peaks collapsing at $z>2$ quickly merge together within groups and their star formation is truncated when their gas is used up. Thus in the hierarchical clustering picture, galaxies that form at high redshifts are in some sense preselected to end up as cluster elliptical galaxies. What is shown here, is that this process can work in detail.

The second conclusion is that bright elliptical galaxies in the field are intrinsically different to cluster ellipticals. These galaxies have undergone recent merging and star formation. It is only because we "catch them at the right time" that we observe them as elliptical systems. In a few gigayears, they will have grown new disks and have turned back into spirals. Finally, I derive predictions for the colour distributions of elliptical galaxies in clusters at high redshift. The scatter is predicted to increase very substantially at redshifts greater than 1 .

\section{References}

Bardeen, J.M., Bond, J.R., Kaiser, N., Szalay, A.S., 1986, APJ, 304, 15

Barnes, J.E., Hernquist,L. 1992, Ann.Rev.Astr.Ap., 30, 705

Bender, R., Burstein, D., Faber, S.M. 1993, ApJ, 411, 153 (BBF)

Bond, J.R., Cole, S., Efstathiou, G., Kaiser, N. 1991, ApJ, 379, 440

Bower, R. 1991, MNRAS, 248, 332

Bower, R., Lucey, J.R., Ellis, R.S. 1992, MNRAS, 254, 601

Bruzual, G., Charlot, S. 1993, ApJ, 405, 538

Charlot, S., Silk, J., 1994, APJ, 405, 538

Ellis, R.S., 1995, in Unsolved Problems in Astrophysics, eds Ostriker, J.P. \& Bahcall, J.N., Princeton, in press

Griffiths, R.E. et al 1995, ApJ, in press

Kauffmann, G., White, S.D.M. 1993, MNRAS, 261, 921

Kauffmann, G., White, S.D.M.,Guiderdoni, B. 1993, MNRAS, 264, 201 (KWG)

Kauffmann, G. 1995, MNRAS, 274,161

Kennicutt, R.C. 1983, ApJ, 272, 54

Renzini, A. 1995, in Stellar Populations, ed Gilmore, G. \& van der Kruit, P. (Dordrecht:Kluwer), in press

Simien, F., De Vaucouleurs, G. 1986, ApJ, 302, 564

GERHARD: Both you and Carlos Frenk had a baryon density $\Omega_{b}=0.1$ in your models. How much of this is tranformed into stars and how is this constrained?

KAUFFMANN: The total mass of stars that is formed is constrained by requiring that the mass and lumininosity of the central galaxy in a halo of circular velocity $220 \mathrm{~km} / \mathrm{s}$ be equal to that of our own Milky Way. The total $\Omega$ in stars is about $0.01-0.02$. 


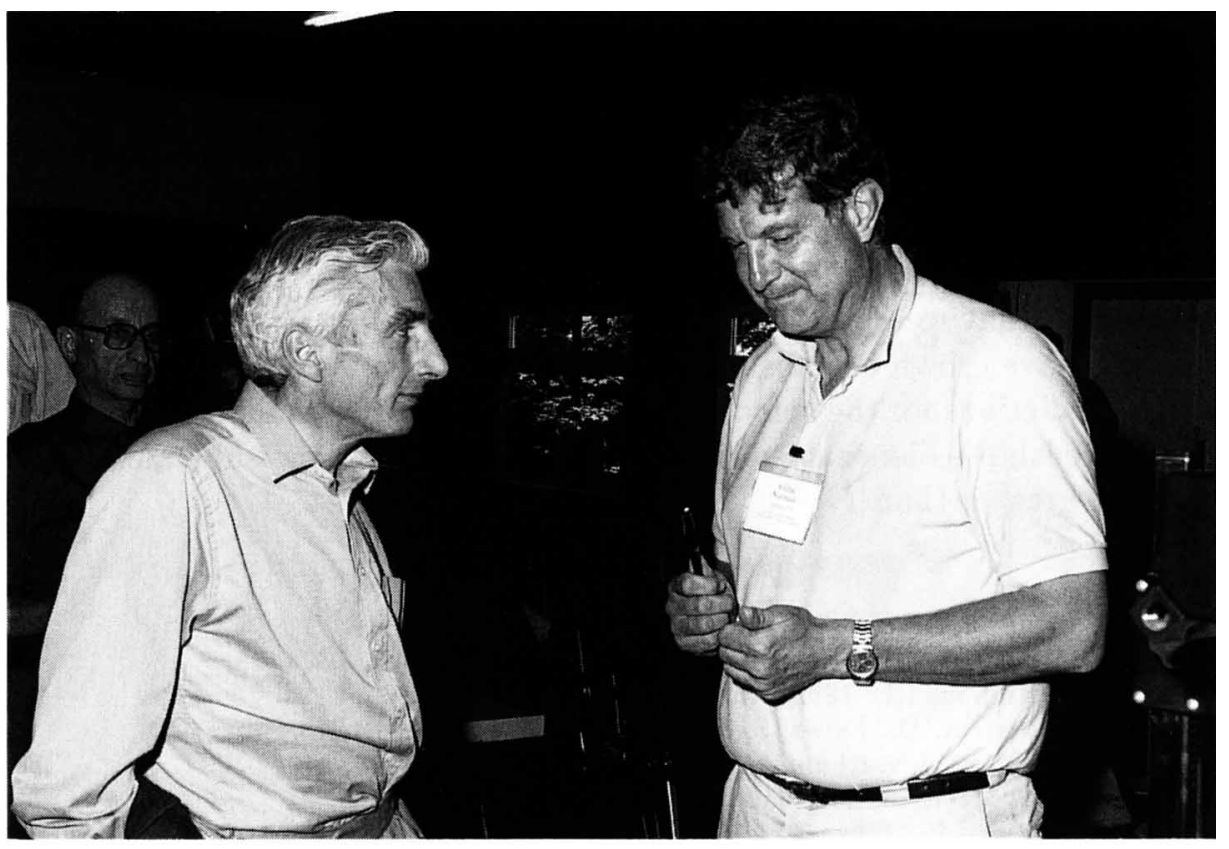

Martin Rees and Colin Norman 